\title{
L'auteur, le symbole et le hasard (Mallarmé, Borges, Cortázar, Calvino)
}

\section{Micéala Symington}

\section{OpenEdition}

12 Journals

\section{Édition électronique}

URL : http://journals.openedition.org/recherchestravaux/1277

DOI : 10.4000/recherchestravaux.1277

ISSN : 1969-6434

Éditeur

UGA Éditions/Université Grenoble Alpes

\section{Édition imprimée}

Date de publication : 15 mai 2004

Pagination : 129-140

ISBN : 2-9518254-3-9

ISSN : 0151-1874

Référence électronique

Micéala Symington, «L'auteur, le symbole et le hasard (Mallarmé, Borges, Cortázar, Calvino) »,

Recherches \& Travaux [En ligne], 64 | 2004, mis en ligne le 20 mai 2019, consulté le 08 septembre 2020.

URL : http://journals.openedition.org/recherchestravaux/1277 ; DOI : https://doi.org/10.4000/ recherchestravaux.1277 


\section{L'auteur, le symbole et le hasard (Mallarmé, Borges, Cortázar, Calvino)}

Pour des raisons et sous des formes différentes, Mallarmé, Borges, Cortázar et Calvino mettent en place une figure de l'auteur qui ne cherche à se construire que dans le paradoxe. À travers l'étude de la question du hasard, c'est un véritable parcours de la modernité qui se dessine, parcours où une interrogation sur l'identité, le rôle ou le statut de l'auteur semble figurer au premier plan. Car l'étude de l'auteur dans ou à travers son texte permet de dégager différentes conceptions de la littérature.

A l'époque symboliste, la place de l'auteur dans le texte se trouve remise en question, et c'est peut-être Mallarmé qui pousse ce questionnement à son point ultime. Par sa disposition typographique entre autres, son poème $U_{n}$ coup de dés jamais n'abolira le hasard" (I897) suggère l'acte de lecture ${ }^{2}$, si bien que le poème peut se lire comme la mise en scène de la relation problématique entre un auteur, absent, et le lecteur, paradoxalement seul présent par son regard, dans le texte. L'œuvre de Borges peut se concevoir d'une certaine manière comme une réponse à cette tentative. La nouvelle intitulée «Pierre Menard, autor del Quijote» (Ficciones, 1944) peut être lue comme une allégorie de l'abolition du hasard dans l'écriture. Dans Rayuela (1963), Julio Cortázar instaure une relation particulière entre auteur et lecteur en proposant un jeu où au moins deux possibilités de lecture du roman sont possibles:

I. Ce texte paraît d'abord en mai 1897 dans une revue intitulée Cosmopolis. In CEuvres complettes de Mallarmé, édition établie et annotée par Henri Mondor et G. Jean-Aubry. Paris, Gallimard, coll. «Bibliothèque de la Pléiade », 1945 (désormais OC), p. 457-477.

2. Voir David Scott, Pictorialist Poetics. Poetry and the Visual Arts in Nineteenth Century France, Cambridge University Press, 1988, p. 138 sqq. 
altérer l'ordre de lecture des chapitres revient à altérer l'intrigue. La figure du lecteur, là encore prégnante par rapport à celle de l'auteur, se confond finalement avec ce dernier, dans la mesure où c'est la lecture qui achève la construction du texte. La question du hasard se trouve également au cœur des préoccupations d'Italo Calvino qui, dans Se una notte d'inverno un viaggiatore (1979), construit son texte autour de la mise en scène de l'acte de lecture.

À propos du poème de Mallarmé, Un coup de dés, Paul Valéry fait cette remarque:

[...] Mallarmé, m'ayant lu le plus uniment du monde son Coup de dés, comme simple préparation à une plus grande surprise, me fit enfin considérer le dispositif. Il me sembla de voir la figure d'une pensée, pour la première fois placée dans notre espace $[\ldots]^{3}$.

Valéry situe l'innovation de Mallarmé sur plusieurs plans: d'abord dans la disposition du texte (l'aspect concret et visuel du poème) qui, sans être simple représentation ou calligramme, participe pleinement à la création du sens; ensuite dans la mesure où il s'agit de "la figure d'une pensée ", c'est-àdire le reflet dans cette disposition d'une attitude de l'esprit ${ }^{4}$; et enfin en ce que cette structure est "placée dans notre espace ": Mallarmé entre dans une nouvelle relation avec le lecteur. Le poème, "pure création de l'esprit", renferme, dans sa structure même, sa propre identité physique en tant qu'objet, et anticipe sur sa lecture. D'une certaine façon, Un coup de dés représente donc un dépassement de l'idée de la "disparition élocutoire du poète" ". La remise en question du rôle de l'œuvre de l'art comme imitation du réel entraînerait un nouveau rapport entre auteur et lecteur.

En effet, c'est à travers le symbole que Mallarmé cherche à révéler un monde invisible, un monde de la suggestion. Mais, justement par l'ouverture qu'il suppose, le symbole propose un nouveau rôle au lecteur. Il ne s'agit plus

3. Paul Valéry, Variété II, Paris, NRF, 1929. Ce passage est tiré du chapitre intitulé le Coup de dés, p. 169-175 (in $O C$ de Mallarmé, p. 1582).

4. Dans une lettre à Camille Mauclair à propos d'Un coup de dés, Mallarmé écrit: "Je crois que toute phrase ou pensée, si elle a un rythme, doit le modeler sur l'objet qu'elle vise et reproduire, jetée à nu, immédiatement, comme jaillie en l'esprit, un peu de l'atcitude de cet objet quant à tout ". Correspondance recueillie, classée et annotée par Henri Mondor avec la collaboration de Jean-Pierre Richard (vol. I); éd. H. Mondor et L. J. Austin (vol. II et III); éd. L. J. Austin (vol. IV-XI, Paris, Gallimard, 1959-85, iI volumes, vol. DX, p. 288.

5. "L'œuvre pure implique la disparition élocutoire du poète, qui cède l'initiative aux mots, par le heurt de leur inégalité mobilisés; ils s'allument de reflets réciproques comme une virtuelle traînée de feux sur des pierreries, remplaçant la respiration perceptible en l'ancien souffle lyrique ou la direction personnelle enthousiaste de la phrase», Mallarmé, "Crise de Vers $n$, in $O C$, p. 366. 
pour lui de comprendre ce qui est désigné, mais d'ouvrir son esprit à une suggestivité symbolique. C'est dans Un coup de dés que la beauté de l'art, qui est figurative, rejoint le symbole poétique. Le symbole, qui suscite la réflexion et représente une forme de beauté moins transparente car moins immédiate, devient aussi, dans ce poème, figuration de la beauté visuelle. Nous pouvons par conséquent considérer que c'est dans ce poème que les idéaux du mouvement symboliste sont le mieux incarnés. En effet, la symbolisation fonctionne sur plusieurs plans (visuel, sonore, cognitif) dans un dépassement radical de la simple représentation.

La confrontation du poème avec la nouvelle de Borges, "Pierre Menard, autor del Quijote", publiée une cinquantaine d'années plus tard, ne représente peut-être pas une comparaison évidente. Mais, en réalité, la nouvelle de Borges ne peut se lire que par rapport aux acquis du symbolisme. Car il s'agit bien d'une remise en question de ces acquis, un retour sur la «crise de la représentation ". Et la question posée par Mallarmé de la "disparition élocutoire du poète, qui cède l'initiative aux mots ${ }^{6}$ " est abordée ici de façon radicale?.

La nouvelle affirme d'emblée un retour au réel, et au visible: "La obra visible que ha dejado este novelista es de fácil y breve enumeración ${ }^{8}$ ". Dès la première phrase, le réel occupe donc une place importante. Le narrateur s'érige en critique offensé par les inexactitudes du travail de Madame Henri Bachelier ("imperdonables las omisiones y adiciones perpetradas $\left[. . .{ }^{9}{ }^{n}\right.$ ) et ce faisant souligne l'importance du détail, du réel. L'énumération qui suit des archives de Menard produit une véritable confusion des genres: la fiction imite la critique, le domaine de l'imagination s'approprie les ressorts de

\section{Ibid.}

7. En ce qui concerne Borges, le rapport entre l'auteur et son texte a déjà été examiné du point de vue de la psychocritique, notamment par Emir Rodríguez Monegal qui, dans "Borges and Derrida. Apothecaries» (in Borges and His Successors. The Borgesian Impact on Literature and the Arts, edited by Edna Aizenberg, Columbia and London, University of Missouri Press, 1990, p. 128-138), p. 129, affirme: "I developed the theory that Borges had preferred reading to writing as a way of denying auchorship, that is, of admitting to the paternity of his works": "J'ai développé la théorie selon laquelle Borges avait préféré la lecture à l'écriture comme moyen de nier sa qualité d'auteur, c'est-à-dire de reconnâtre la paternité de ses œuvres" (notre traduction).

8. Borges, "Pierre Menard, autor del Quijoten (Ficciones, 1944) in Obras completas vol. I (1923-1949), Barcelona, Maria Kodama y Emecé Editores, 1989, P. 444. Nous urilisons la traduction de P. Verdevoye, Ibarra et Roger Caillois: «Pierre Menard, auteur du Quichottew in Fictions Paris, Gallimard (nouvelle édition augmentée), 1983 : «L'ouvre visible qu'a laissée ce romancier peut être facilement et brièvement passée en revuew (p. 4I).

9. Ibid.: «Impardonnables par conséquent sont les omissions et les additions perpétrées $[\ldots]$ " (op. cit., p. 4r). 
l'analyse érudite et minutieuse. Les philosophes et écrivains qui sont cités (Leibniz, Russell, Descartes, Valéry, par exemple) n'appartiennent pas au domaine de la fiction et les revues mentionnées (par exemple, Revue des langues romanes, $N R F$ ) existent réellement. Tout semble concourir à un ancrage ferme dans la réalité. Pourtant, en même temps, Borges semble se moquer des théories qui font du roman un laboratoire pour l'étude du réel ${ }^{10}$. Ce qui compte enfin n'est pas de savoir si Menard existait réellement ou pas, mais de s'interroger sur la spécificité de l'œuvre.

Et c'est à travers la question de la spécificité absolue de l'œuvre littéraire que nous retrouvons la problématique du hasard évoquée d'abord par Mallarmé ("un coup de dés jamais n'abolira le hasard"). Reproduire "palabra por palabra y línea por línea ${ }^{\mathrm{II}}$ " l'œuvre d'un autre, tâche que s'était donnée, apparemment avec succès, le héros éponyme de l'histoire de Borges, semble représenter la preuve d'une certaine perfection ou nécessité narrative: un refus du hasard qui tourmentait Mallarmé et dont il est question dans $U_{n}$ coup de dés.

En effet, le poème de Mallarmé traite explicitement et implicitement du problème du hasard ${ }^{\mathrm{I} 2}$ : la tâche du poète consiste en la tentative d'élimination du hasard, pour produire l'œuvre parfaite où le hasard du langage est vaincu par la nécessité poétique. À propos de cette question, Paul Valéry s'appuie sur son expérience directe de l'attitude de Mallarmé envers son travail pour dénoncer la démarche consistant à faire d'Un coup de dés une œuvre théâtrale:

Si elles [les personnes qui veulent metrre le Coup de dés sur scène] eussent, le moins du monde, connu Mallarmé vivant, et qu'elles eussent, comme moimême, entendu ce grand homme discuter (au sens presque de l'algèbre) les moindres détails de position du système verbal et visuel qu'il avait construit, si elles l'eussent assisté, vérifiant minutieusement le montage de cette figure en qui devaient se composer le simultané de la vision avec le successif de la parole, comme si un équilibre très délicat eût dépendu de ces précisions, je les assure que

Io. Voir par exemple Lois Oppenheim, Intentionality and Intersubjectivity. $A$ Phenomenological Study of Butor's La Modification, Lexington, Kentucky, French Forum Publishers, 1980, p. 14: «[...] Butor's conception of the novel as a laboratory for the study of reality may be likened to that of the phenomenologist ": "[...] la conception du roman de Butor comme laboratoire pour l'étude de la réalité peut être assimilée à la conception phénoménologique".

II. Borges, "Pierre Menard, autor del Quijote" in Obras completas, op. cit., p. 446: "mot à mot et ligne à ligne" (Fictions, op. cit., p. 45).

12. Sur le problème du hasard, voir notamment l'analyse très systématique de Bertrand Marchal, Lecture de Mallarmé. Poessies - Igitur - Le coup de dés, Paris, Librairie José Corti, I985, p. $27 \mathrm{I}-288$. 
l'idée, jamais, ne leur serait venue d'abolir, au moyen d'interprètes, tout ce profond calcul par le hasard! ${ }^{13}$

A la différence de la pièce de théâtre, qui atteint sa plus haute expression dans la mise en scène, et du roman réaliste, qu'une lecture à haute voix rend pleinement compréhensible, Un coup de dés exige une appréciation de l'œuvre à la fois comme évocation d'une réalité indépendante des signes sur la page et comme objet dont la page représente toute la réalité. Bien que cette œuvre affirme explicitement l'impossibilité de vaincre le hasard dans la réalisation de l'œuvre parfaite, tout concourt dans le poème à éliminer autant que possible le hasard. Transformer $U n$ coup de dés en pièce de théâtre revient donc à introduire dans l'œuvre une part de hasard qui n'existe pas dans l'œuvre telle que la conçoit Mallarmé.

Dans Un coup de dés, tout est symbole: y compris la sonorité, la typographie, les blancs. Comme l'a dit Valéry, Mallarmé essaie ici "d'élever enfin une page à la puissance du ciel étoilé! " 4 "Cette convergence entre les dimensions acoustique et graphique dans la production de sens est particulièrement évidente à la fin d'Un coup de dés, où le mouvement des dés qui roulent doucement avant de s'arrêter totalement est suggéré par la disposition en diagonale des mots qui forment des pas sur la page. Le bruit léger du dé est également présent dans la sonorité en [ā] des participes présents ("veillant», "doutant", "roulant", "brillant", "méditant") dont la répétition produit un léger effet hypnotique. Le changement de rythme suggéré par la liaison avec "et" ("brillant et méditant") évoque un double mouvement rebondissant du dé, qui produit donc un double son. Le lien entre mouvement et son, forme graphique et forme acoustique, est donc plus que jamais présent. Le fait que ce soit dans Un coup de dés, le poème qui s'approche le plus de la perfection, selon le rêve mallarméen d'une synthèse des arts, que Mallarmé admet l'impossibilité de l'œuvre parfaite ("un coup de dés jamais n'abolira le hasard") semble donc paradoxal.

Si nous examinons l'œuvre de Borges dans cette perspective, nous pouvons donc considérer qu'il s'agit d'une réflexion à propos de la question du hasard: reproduire l'œuvre d'un autre, est-ce preuve d'une perfection artistique ou nécessité narrative? Le hasard peut-il être vaincu?

En effet, si nous reprenons les théories intentionalistes, dans lesquelles l'œuvre d'art est envisagée comme manifestation de l'intention de l'artiste, il s'agit d'imaginer la possibilité d'un rapport identique que deux auteurs dis-

13. Paul Valéry, Varieté, in op. cit., p. 625.

I4. Ibid., p. 626. L'italique est de Valéry. 
tincts pourraient entretenir avec le monde. C'est cette thèse qui est développée par Raphaël Lellouche dans Borges ou l'hypothèse de l'auteur. Pour lui, l'idée d'une répétition pure, par Menard, de l'œuvre de Cervantès suppose un même rapport au monde:

Menard ne renonce pas à être Menard. Il écarte la voie de l'identification, de "l'être" qui est de s'approprier dynamiquement, dit Lacan, les propriétés de l'objet d'identification. [...]

En fait il y a bien une solution, une seule, pour que ce fût possible: c'est que l'expérience "particulière" de Menard inclue, mais infiniment, celle de Cervantès ${ }^{15}$.

R. Lellouche s'attache à trouver une réponse vraisemblable à l'énigme posée par l'œuvre de Menard. Il pose donc la question de ce qui est métaphysiquement possible. Mais au-delà de l'hypothèse de la répétition de la relation entre l'auteur et monde, le projet de Menard représente une réponse radicale au Coup de dés de Mallarmé ${ }^{16}$ : il s'agit de l'abolition du hasard, donc de la réalisation de l'idéal mallarméen. S'il est possible pour un auteur de reproduire le texte d'un autre, c'est que chaque mot de ce texte est justifié et nécessaire. La nécessité poétique, la perfection du geste littéraire n'est plus à prouver, le hasard peut alors être vaincu.

La question du hasard dans l'œuvre littéraire, l'idée selon laquelle l'œuvre puisse constituer une configuration parfaite et immuable est une question qui obsède Borges. À propos de Cortázar, par exemple, il affirme: «El estilo ne parece cuidado, pero cada palabra ha sido elegida. Nadie puede contar el argumento de un texto de Cortázar; cada texto consta de determinadas palabras en un determinado orden. Si tratamos de resumirlo verificamos que algo precioso se ha perdido ${ }^{17}$ ». Borges prend ici le rôle du critique littéraire; lecteur de Cortázar. Sa position est semblable à celle de son personnage, Pierre Menard, à l'égard de Cervantès et l'obsession de la perfection littéraire se trouve également reproduite ${ }^{18}$. Cette notion semble d'autant plus para-

I5. Borges ou l'hypothèse de l'auteur, Paris, Balland, 1989, p. 194.

16. Dans la liste des ceuvres de Menard figurent « un sonnet symboliste * ainsi que des travaux sur Paul Valéry. Borges semble vouloir conférer ainsi à son personnage un intérêt particulier pour les thèses symbolistes.

17. Borges, Biblioteca Personal, Madrid, Alianza Editorial, 1988, p. 9-10: “Julio Cortázar Cuentos", p. 10: "Le style ne semble pas soigné, mais chaque mot a été choisi. Personne ne peut raconter l'argument d'un texte de Cortázar; chaque texte se compose de mots déterminés dans un ordre déterminé. Si nous essayons de le résumer, nous constatons que quelque chose de précieux a été perdu " (Notre traduction).

I8. A propos de "Pierre Menard, autor del Quijote ", R. Lellouche écrit: "La tâche surhumaine que s'impose Menard est à l'écriture ce que la Kabbale est à la lecture. [...] Soit une lecture qui part du principe qu'il n'y a rien dans le texte lu qui ne soit justifié, rien qui n'ait sa 
doxale que Cortázar, lui, semble au contraire s'enfoncer dans le hasard de la fiction. Au début de Rayuela, il interdit une lecture unique:

A su manera este libro es muchos libros, pero sobre todo es dos libros.

El primero se deja leer en la forma corriente, y termina en el capítulo 56 , al pie del cual hay tres vistosas estrellitas que equivalen a la palabra Fin. Por consiguiente, el lector prescindirá sin remordimientos de lo que sigue.

El segundo se deja leer empezando por el capítulo 73 y siguiendo luego en el orden que se indica al pie de cada capítulo. En caso de confusión u olvido, bastará consultar la lista siguiente: $73-\mathrm{I}-2-116-3-84-4[\ldots]^{19}$.

Rayuela se lit d'au moins deux façons. En proposant au lecteur le choix de l'ordre des chapitres, Cortázar intègre la notion de hasard dans le tissu même de l'œuvre. Le rapport entre auteur et lecteur intègre forcément la notion de liberté: l'auteur ne peut pas savoir quelle version sera choisie par son lecteur. Dans la tension entre la "nécessité", ou déterminisme littéraire ${ }^{20}$ ("chaque texte se compose de mots déterminés dans un ordre déterminé") qui pour Borges caractérise l'œuvre de Cortázar, et la remise en question de cette nécessité par Cortázar lui-même, se trouve résumé le paradoxe du littéraire.

Dans Rayuela, Cortázar instaure une relation particulière entre auteur et lecteur en proposant ce jeu où altérer l'ordre de lecture des chapitres revient à altérer l'intrigue. Les chapitres "non essentiels" occupent en réalité plusieurs fonctions : ils servent à subvertir la narration principale, ou bien à multiplier ses résonances ${ }^{21}$. La figure du lecteur se confond finalement avec celle de l'auteur, dans la mesure où c'est la lecture qui achève la construction du

raison précise, une lecture qui n'admet aucun hasard "(Borges ou l'hypothese de l'auteur, op. cit., p. 199 et 200). C'est cette absence de hasard que Borges souligne à propos de l'écriture de Cortázar.

19. Julio Cortázar, Rayuela. Prólogo y Cronologła de Jaime Alazraki, Caracas (Venezuela), Biblioteca Ayacucho, 1988, p. 3: «A sa façon, ce livre est plusieurs livres mais en particulier deux livres. Le lecteur est invité à choisir entre les deux possibilités suivantes: le premier livre se lit comme se lisent les livres d'habitude et il finit au chapitre 56 , là où trois jolies petites étoiles équivalent au mot Fin. Après quoi, le lecteur peut laisser tomber sans remords ce qui suit. Le deuxième livre se lit en commençant au chapitre 73 et en continuant la lecture dans l'ordre indiqué a la fin de chaque chapitre. En cas d'incertitude ou d'oubli, il suffira de consulter la liste ci-dessous : 73-I-2-I16-3-84-4 [...] * (Marelle, traduit de l'espagnol par Laure Guille et Françoise Rosset, Paris, Gallimard, 1966, p. 7).

20. L'analyse de Borges n'est pas tout à fait représentative de celle de la critique en général. Carlos Henderson (Estudios sobre la poetica de Rayuela. Madrid, Editorial Pliegos, 1995) par exemple, s'attache au contraire à montrer l'aspect multiple, "carnavalesque", de l'cuvre de Cortázar (p. 84) : "En efecto, en Rayuela no hay palabra última, unívoca : "En effet, dans Rayuela, il n'y a pas de mot ultime, univoque" (notre traduction).

2I. Steven Boldy propose une analyse de la fonction de ces chapitres dans son ouvrage The Novels of Julio Cortázar, Cambridge, Cambridge University Press, 1980. 
texte. L'auteur est présent dans le texte, mais sa fonction auctoriale est remise en question et le choix proposé au lecteur dans l'ordre des chapitres s'oppose à une conception univoque, immuable de l'œuvre. Le titre de l'ouvrage, Rayuela (marelle) suggère que la relation entre auteur et lecteur s'apparentera à un jeu où le rôle traditionnel de chacun se trouvera ébranlé 22 .

Le jeu et le hasard se trouvent également au cœur des préoccupations d'Italo Calvino qui, dans Se une notte d'inverno un viaggiatore (1979), construit son texte autour de la mise en scène de l'acte de lecture. Mais ce faisant, il place l'auteur, de ce fait lui aussi véritable personnage de la narration, en position de locuteur: le texte même intègre la contingence de sa lecture et l'auteur est figuré à travers un dialogue avec son lecteur. Dès la première phrase du roman, cette relation est instaurée: "Stai per cominciare a leggere il nuovo romanzo Se una notte d'inverno un viaggiatore di Italo Calvino. Rilassati. Raccogliti. Allontana da te ogni altro pensiero. Lascia che il mondo che ti circonda sfumi nell'indistinto. La porta è meglio chiuderla $[\ldots]^{23}$ ”. Le narrateur/auteur s'adresse directement au lecteur. Monde fictionnel (le monde du narrateur/auteur) et monde réel (le monde du lecteur) deviennent un: la voix narrative se permet une intrusion dans l'intimité du lecteur («il vaut mieux fermer la porte»). Les regards sont inversés: le lecteur ne peut pas occuper ici son rôle de spectateur des événements du monde fictionnel ainsi créé, c'est lui qui se trouve l'objet du regard du narrateur/auteur. Calvino se plaît ainsi à jouer des règles du jeu romanesque, comme il l'affirme dans un essai intitulé "Le roman comme spectacle»:

[...] si nous connaissons les «règles» du jeu romanesque, nous pourrons construire des romans "artificiels" nés en laboratoire, nous pourrons jouer au roman comme on joue aux échecs, avec une absolue loyauté, en rétablissant une communication entre l'écrivain, pleinement conscient des mécanismes dont il est en train de faire usage, et le lecteur qui accepte le jeu parce qu'il connaît les règles et sait qu'il ne peut plus être pris au piège ${ }^{24}$.

22. Voir à ce propos l'analyse de Maria Dolores Blanco Arnejo, La novela lídica experimental de Julio Cortázar. Madrid, Editorial Pliegos, 1996.

23. Italo Calvino, Se una notte d'inverno un viaggiatore, in Romanzi e Racconti. Edizione diretta da Claudio Milanini a cura di Mario Barenghi e Bruno Falcetto, Milan, Palomar, 1992, vol. II, p. 613. Si par une nuit d'biver un voyageur. Traduit de l'italien par Danièle Sallenave et François Wahl, Paris, éditions du Seuil, 1995, p. 9: «Tu vas commencer le nouveau roman d'Italo Calvino, Si par une nuit d'hiver un voyageur. Détends-toi. Concentre-toi. Écarte de toi toute autre pensée. Laisse le monde qui t'entoure s'estomper dans le vague; la porte, il vaut mieux la fermer [...]".

24. Calvino, "Le Roman comme spectacle" in La Machine littéraire, traduit par Michel Orcel et François Wahl, Paris, Seuil, 1984, p. I56. 
L'idée du jeu, que nous retrouvons également chez Cortázar, devient une façon de réclamer le hasard: au lieu de chercher à l'éliminer, Calvino et Borges acceptent d'intégrer cette notion à leur narration, sachant que «le roman finira, même s'il est pratiqué ironiquement, par nous bouleverser malgré nous ${ }^{25}$ ", comme l'affirme Calvino.

Les dix incipit qui forment le roman de Calvino correspondent donc à dix possibilités de roman : le roman se place dans une situation de balancement entre la nécessité romanesque (une abolition du hasard) et les différentes réalisations (les dix débuts de roman), qui deviennent la preuve de l'arbitraire de la narration. Selon Teresa de Lauretis, la réflexivité et le questionnement représentent la véritable intrigue de la nouvelle de Calvino:

In short, this is a text about textuality, a piece of writing about the process of writing; and we are never for a moment allowed to forget that we are, at that very moment, reading it. [...] It tells us how we read, what we do while reading, what we want as readers, as well as what the writer wants, how he writes, what he does while writing and so forth ${ }^{26}$.

Le roman de Calvino opère donc subtilement un changement de focalisation ${ }^{27}$. L'intrigue et les personnages se trouvent relégués au second plan: c'est la nouvelle relation entre auteur et lecteur qui se trouve explorée ici ${ }^{28}$. Mais cette nouvelle relation, qui intègre la possibilité de multiples lectures, écarte forcément la notion d'œuvre absolue.

En effet, si le personnage de Borges, Pierre Menard, réalise l'idéal mallarméen d'abolition du hasard, cette réalisation produit une lecture qui est le contraire d'une lecture symbolique, c'est-à-dire le contraire d'une lecture qui admet la possibilité d'une évocation libre. L'abolition du hasard représenterait à cet égard une impasse du roman.

\section{Ibid.}

26. Teresa de Lauretis, "Reading the (Post) Modern Text: If on a winter's night a traveler", in Calvino Revisited. Edited by Franco Ricci. University of Toronto, Italian Studies 2, Toronto, Dovehouse Editions Inc., 1989, p. 135: "Bref, c'est un texte sur la textualité, un morceau d'écriture sur le processus d'écrire; et l'on ne nous permet jamais, même pas une seconde, d'oublier que nous sommes à ce moment même en train de le lire. [...] Il nous dit comment nous lisons, ce que nous faisons en lisant, ce que nous voulons, nous lecteurs, ainsi que ce que veut l'écrivain, comment il écrit, ce qu'il fait quand il écrit et ainsi de suite ".

27. Voir à ce propos l'analyse de Jean-Paul Manganaro, Italo Calvino. Romancier et conteur, Paris, Editions du Seuil, 2000. Selon J.-P. Manganaro, par exemple (p. 132): «Ayant fait le tour de ces ensembles qui racontent cosmos, château, taverne et ville, il ne restait plus à Calvino qu'à élucider les procédés créatifs des ceuvres en tant que système de transmission de signes entre auteur et lecteur\%.

28. Selon Jerry Varsava, il s'agit d'une problématisation de l'acte de lecture dans une célébration du plagiat. "The Last Fictions. Calvino's Borgesian Odysseys" in Borges and His Successors, The Borgesian Impact on Literature and the Arts, op. cit., p. 183-199. 
Par ailleurs, l'argument est réversible: si chaque texte peut avoir plusieurs auteurs (l'auteur de Don Quichotte peut être Cervantès et Menard), alors les possibilités de lecture se démultiplient et le contexte acquiert une importance démesurée. Borges joue de cette idée: «También es vívido el contraste de los estílos. El estilo arcaizante de Menard - extranjero al fin - adolece de alguna afectación. No así el del precursor, que maneja con desenfado el espaniol corriente de su época ${ }^{29}$ ». La contextualisation, qui ouvre la voie à d'innombrables lectures, représente le retour du hasard. En fin de compte, la répétition parfaite du Quichotte est impossible, car l'œuvre, même autotélique, n'échappe pas au contexte.

Dans Rayuela, une réflexion sur la notion même de choix est amorcée et cette question est reliée à la notion de vérité:

El solo hecho de interrogarse sobre la posible elección vicia y enturbia lo elegible. [...] Parecería que una elección no puede ser dialéctica, que su planteo la empobrece, es decir la falsea, es decir la transforma en otra cosa. Entre el Yin y el Yang, ¿cuántos eones? [...] ; Pero de qué nos sirve la verdad tranquiliza al propietario honesto? Nuestra verdad posible tiene que ser invencion, es decir escritura, literatura, pintura, escultura, agricultura, piscicultura, todas las turas de este mundo ${ }^{30}$.

La question "Entre le Yin et le Yang, combien d'éons?" souligne l'impossibilité du choix simple. A partir du moment où un choix s'annonce, les possibilités se multiplient ("un dilemme ne peut être dialectique»). En proposant deux façons de lire son roman, Cortázar sait qu'il ouvre la voie à une multiplicité de lectures, et c'est ce qu'il déclare au commencement du roman: "A sa façon, ce livre est plusieurs livres mais en particulier deux livres. » Si nous suivons la logique de Cortázar, c'est justement parce que le livre est deux livres qu'il est "plusieurs livres». Dans cette impasse, la seule "vérité" demeure la création ("Notre seule vérité possible doit être invention"). Autrement dit, plus le lecteur ou l'auteur aura de possibilités inventives, créatives, plus il s'approchera de la vérité. Cette conception de la litté-

29. Borges, "Pierre Menard, autor del Quijote" in Obras completas, op. cit., p. 449. Traduction: Fictions, op. cit., p. so: «le contraste entre les deux styles est également vif. Le style archaïsant de Menard - tout compte fait étranger - pèche par quelque affectation. II n'en est pas de même pour son précurseur, qui manie avec aisance l'espagnol de son époquè.

30. Rayuela, op. cit., ch. 73, p. 325-6: «Le seul fait de s'interroger sur la possibilité du choix altère et trouble ce qu'on choisit. Il semblerait qu'un dilemme ne puisse être dialectique, que le seul fait de poser le problème l'appauvrisse, c'est-à-dire le fausse, c'est-à-dire le transforme en quelque chose d'autre. Entre le Yin et le Yang, combien d'éons? [...] Mais à quoi nous sert la vérité qui rassure l'honnête propriétaire? Notre seule vérité possible doit être invention, c'est-à-dire écriture, littérature, peinture, sculpture, agriculture, pisciculture, toutes les "tures" de ce monde. (Marelle, op. cit, p, 398). 
rature embrasse le hasard: c'est à travers le hasard que l'auteur/lecteur s'approche de la vérité du texte.

Mais, paradoxalement, cette conception du hasard peut être rapprochée de la théorie du symbole ${ }^{31}$. Il s'agit dans les deux cas de la recherche du lien fondamental et universel entre monde visible et monde invisible atteint grâce à une ouverture suggestive ${ }^{32}$. Cortázar lui-même se sert d'une comparaison avec le symbole pour proposer sa définition de l'œuvre comme « una manifestación consubstancial de su ser, no un mediatizado símbolo estético [... ${ }^{33}$ ". Les romanciers du $\mathrm{XX}^{\mathrm{c}}$ siècle en viennent donc à rejeter le symbole pur (qui tend à effacer l'auteur), ainsi que l'idéal d'une œuvre absolue où le hasard n'a pas de place. Ils veulent, au contraire, privilégier une autre façon de symboliser, plus structurelle, qui s'appuie sur cette relation entre auteur et lecteur, afin de permettre un accès au monde invisible. A propos des Villes invisibles, Calvino écrit:

[...] j'ai construit une structure à facettes où chaque court texte côtoyant le voisin sans que leur rapport implique un rapport causal ou hiérarchique se trouve pris dans un réseau qui permet de tracer des parcours multiples et de tirer des conclusions ramifiées et plurielles ${ }^{34}$.

Dans les Villes invisibles, les différents textes prennent place dans un réseau qui permet des parcours multiples, ramifiés, pluriels. La contextualisation représente un moyen de dépassement du texte individuel, de l'unicité de sens. Nous avons déjà cité "Crise de Vers", texte où Mallarmé analyse le fonctionnement symbolique: les mots "s'allument de reflets réciproques comme une virtuelle traînée de feux sur des pierreries ${ }^{35}$ ». C'est la place du

31. Selon Walter Benjamin, le symbole repose sur *la liaison intrinsèque entre la forme et le contenu * (Origine du drame baroque allemand, traduit de l'allemand [Ursprung des deutschen Trauerspiels, dans les ceuvres complètes de Walter Benjamin en 6 vol., éd. par Rolf Tiedemann et Hermann Schweppenhäuser, avec la collaboration de Theodor W. Adorno et Gershom Sholem, t. I, I, Francfort-sur-le-Main, Suhrkamp, 1974] par Sibylle Muller (avec le concours de André Hirt), préface de Irving Wohlfarth, Paris, Flammarion, 1985, p. 172) qui fait que le symbole est tout à la fois l'Idée et son support matériel, dans une dialectique qui refuse la distinction du matériel et du spirituel, du limité et de l'illimité.

32. Charles Morice, dans sa réponse à Jules Huret, souligne le lien indissociable entre suggestion et fiction du poème; il est pour lui ce qui constitue le symbole, compris comme "le mélange des objets qui ont éveillé nos sentiments et de notre âme en une fiction. Le moyen, c'est la suggestion: il s'agit de donner aux gens le souvenir de quelque chose qu'ils n'ont jamais vu " (Enquête sur l'évolution litteraire. Paris, Charpentier, 1891, p. 85).

33. Cortázar, "La crisis del culto al libro ", in Obra critica I. Edición de Saúl Yurkievich, Madrid, Alfaguara, 1994, p. 40: "une manifestation consubstantielle de son être, non pas un symbole esthétique médiatisé" (notre traduction).

34. Calvino, Leçons américaines, Paris, Gallimard, 1988. Traduit par Y. Hersant, p. II8.

35. Mallarmé, "Crise de Vers", in $O C$, p. 366 (déjà cité). 
mot dans un contexte qui permet le dépassement poétique et symbolique, qui fait que le mot cesse d'être simple signe, pour devenir " une virtuelle trâ̂née de feux". En effet, le lien entre l'entreprise de Borges, Calvino, Cortázar et la poétique de Mallarmé semble relever de cette idée de réseau. Mais là où, pour les symbolistes, il s'agit d'un réseau poétique interne qui repose sur le symbole, pour les écrivains post-symbolistes, ce réseau à résonances multiples est un réseau plus large, structurel et textuel. Il n'est possible que dans une structure où le lecteur participe au jeu narratif. Le réseau se construit donc non pas sur une évocation poétique et symbolique, mais sur une présomption de lectures préalables. Calvino a déclaré: "Je voudrais écrire des livres qu'on oublie. C'est quand on oublie un livre qu'il continue à travailler dans notre mémoire ${ }^{36}$ ". Les romans de Calvino exigent un travail de la part du lecteur, une participation particulière à la construction de sens. C'est ainsi que l'auteur cède l'initiative au lecteur: il ne conçoit pas son écriture comme une lutte contre le hasard, car ce hasard représente l'accomplissement de l'œuvre.

Embrasser le hasard dans le roman et affirmer le rôle du lecteur dans la construction du texte aboutit à un véritable retour de l'auteur. C'est peutêtre Cortázar qui affirme le plus clairement la nouvelle position de l'auteur. Le fait qu'il se serve de la comparaison entre sa poétique et le symbolisme pour insister sur cette idée ne relève pas non plus du hasard: "el escritor se siente cada vez más comprometido como persona en la obra que realiza [...] ${ }^{37}$ ». Pour Borges, Cortázar, Calvino, l'auteur ne "cède pas l'initiative" aux mots, il reprend sa place dans l'œuvre grâce à un rapport particulier qui s'établit entre auteur et lecteur. Cette nouvelle relation laisse une plus grande place au hasard: éliminer le hasard ne peut plus représenter l'idéal du geste poétique absolu car le hasard devient un élément essentiel dans un réseau où tout participe à la symbolisation.

36. Calvino, réplique à une question posée dans le film Italo Calvino, série "Un siècle d'écrivains», dirigéc par Bernard Rapp (France 3). Cité par Philippe Daros, «L'image et le mode d'existence de l'objet littéraire dans l'œuvre d'Italo Calvino ", Italo Calvino. Le défi au labyrinthe, Actes de la Journée d'études de Caen, 8 mars 1997, publiés sous la direction de Paolo Grossi et Silvia Fabrizio-Costa, Caen, Presses universitaires de Caen, 1998, p. 98.

37. Cortázar, «La crisis del culto al libro", op. cit., p. 40: "l'écrivain se sent chaque fois plus engagé comme personne dans l'œuvre qu'il réalise ". 